\title{
PH棌ENIX
}

PX No. 204

BNL-68966

\section{RHIC Experimental Results}

\author{
M.J. Tannenbaum \\ Brookhaven National Laboratory, Upton, NY, USA \\ Sixth Workshop on Non-Perturbative QCD \\ American University, Paris, France \\ June 8, 2001 \\ Submitted to proceedings, which will be published in \\ World Scientific Publishing
}

Physics Department

Brookhaven National Laboratory

Operated by

Brookhaven Science Associates

Upton, NY 11973

Under Contract with the United States Department of Energy

Contract Number DE-AC02-98CH10886 


\title{
RHIC EXPERIMENTAL RESULTS
}

\author{
M. J. TANNENBAUM * \\ Brookhaven National Laboratory, Upton, NY 11973-5000, USA \\ E-mail: mjt@bnl.gov
}

\begin{abstract}
A summary of measurements from the first year's run at RHIC is presented. The second year's run at RHIC is presently underway with $\mathrm{Au}+\mathrm{Au}\left(300 \mu \mathrm{b}^{-1}\right)$ and p-p $\left(3.5 \mathrm{pb}^{-1}\right)$ collisions planned at $\sqrt{s_{N N}}=200 \mathrm{GeV}$.
\end{abstract}

\section{RHIC at a Glance}

\subsection{Machine}

In the summer of 2000, RHIC, the Relativistic Heavy Ion Collider at Brookhaven National Laboratory, achieved $\mathrm{Au}+\mathrm{Au}$ collisions. The first collision was recorded on June 12, 2000 at nucleon-nucleon c.m. energy $\sqrt{s_{N N}}=56 \mathrm{GeV}$. The $\mathrm{Au}+\mathrm{Au}$ run continued briefly at $\sqrt{s_{N N}}=56 \mathrm{GeV}$, followed by a more extensive run at $\sqrt{s_{N N}}=130 \mathrm{GeV}$ which yielded integrated luminosity of a few $\mu \mathrm{b}^{-1}$ corresponding to a possible $\sim 15$ million minimum bias events. Requiring an event vertex within the good acceptance of the experiment, reduced the useful luminosity to a several million minimum bias events, around $0.5 \mu \mathrm{b}^{-1}$. However, this being a new machine, siginficant physics could be done even with $\sim 10,000$ minimum bias events as one experiment (PHOBOS) has done at $\sqrt{s_{N N}}=56$ and $130 \mathrm{GeV}$ during the first year's run and already at $\sqrt{s_{N N}}=200 \mathrm{GeV}$ during the present summer 2001 run.

\subsection{Experiments}

For the sake of brevity, suffice it to say that there are 4 experiments taking data at RHIC, two large experiments STAR and PHENIX, and two smaller experiments PHOBOS and BRAHMS. Results will be reported from all 4 experiments with citations to published data or conference proceedings. All the RHIC data are from $\mathrm{Au}+\mathrm{Au}$ collisions at $\sqrt{s_{N N}}=130 \mathrm{GeV}$, except as specifically noted.

\subsection{Global Event Properties - Nuclear geometry and 'impact parameter'.}

For elastic Rutherford scattering of Au nuclei, the impact parameter can be measured from the scattering angle. However, in the highly inelastic region

qcd2001proc: submitted to World Scientific on September 25, 2001

* Research supported by the U.S. Department of Energy, DE-AC02-98CH10886 
of relativistic heavy ion (RHI) collisions, the impact parameter or the equivalent description of the geometry of the collision must be deduced from the overall or global properties of the event. For a collision of two Au nuclei (spheres are a reasonable description) at impact parameter $b$, there is an 'almond' shaped overlap region where the nucleons participate in the collision (participants). The non-participating nucleons which tend to remain at beam (or target) rapidity are considered spectators and can be detected in zero degree calorimeters (ZDC) which are sensitive only to particles at beam or target rapidity. In fixed target experiments, this is straightforward. However, in a collider, the ring magnets sweep away any charged spectator fragments, leaving only free spectator neutrons (or neutral fragments) at zero degrees from the collision axis. All 4 experiments at RHIC have identical sets of ZDC which record any free neutrons remaining at beam rapidity $(|\eta|>6)$ after the $\mathrm{Au}+\mathrm{Au}$ collision. These neutrons are proportional to the number of true projectile and target spectators, hence to the number of participants. ${ }^{1,2}$ Most experiments also use counters in the range roughly $3 \leq|\eta| \leq 4$, called either Beam-Beam or Paddle-Counters, to detect the charged particles emitted from the collision which also provide a complementary measurement of the 'centrality' - the more central the collision(smaller $b$ ), the larger the overlap, the larger the number of participants, the larger the number of particles produced. The centrality is typically quoted as an upper percentile of a reference distribution.

\section{Measurements of Global Quantities}

\subsection{Multiplicity}

The first submission for publication of a physics result from RHIC was by the PHOBOS collaboration on July $19,2000{ }^{3}$. PHOBOS measured the charged-particle multiplicity density, $d n_{c h} /\left.d \eta\right|_{\text {mid }}$, at mid-rapidity for $\mathrm{Au}+\mathrm{Au}$ at $\sqrt{s_{N N}}=56$ and $130 \mathrm{GeV}$ for events with the top 6 percentile of the multiplicity distribution in their reference counter $-6 \%$ centrality, for short. They repeated the feat this year with the submission of their $200 \mathrm{GeV}$ measurement ${ }^{4}$ on August 8, 2001. Figure 1 shows $d n_{c h} /\left.d \eta\right|_{\text {mid }}$ (left) for $\mathrm{Au}+\mathrm{Au}$ central collisions divided by $1 / 2$ the number of participants. At the c.m. energy of the CERN and FERMILAB fixed target programs, the multiplicity in $\mathrm{p}+\mathrm{A}$ collisions compared to $p-p$ (2 participants) was observed ${ }^{5}$ to be proportional to the number of participants $N_{\text {part }}$, i.e. struck or 'wounded nucleons', rather than to the number of possible binary-collisions - the Wounded Nucleon Model (WNM). ${ }^{6}$ If the WNM were to hold in general, then the $\mathrm{Au}+\mathrm{Au}$ results

qcd2001proc: submitted to World Scientific on September 25, 2001 

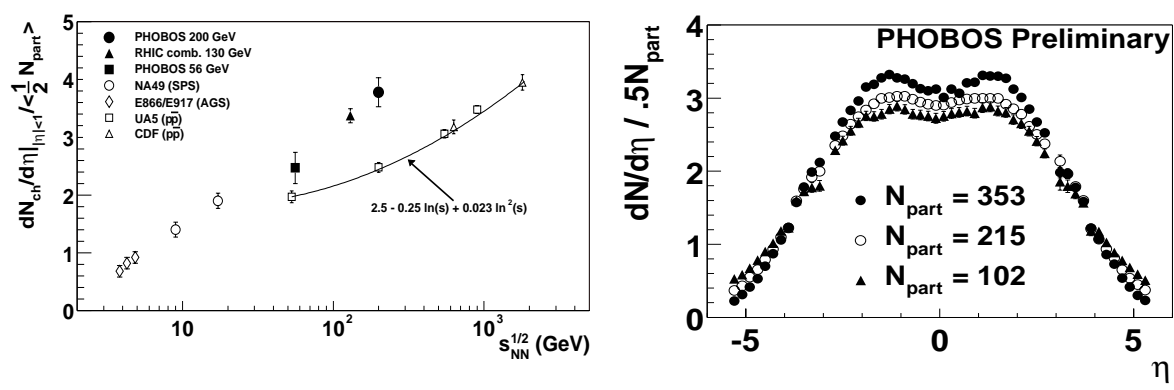

Figure 1. PHOBOS multiplicity. (left) Mid-rapidity $d n_{c h} /\left.d \eta\right|_{\text {mid }}$ per participant pair from RHIC (solid points), lower energy RHI collisions (open points) and $p-\bar{p}$ collisons (open points with line); (right) pseudorapidity distribution of multiplicity per participant pair for 3 different centralities labelled by $N_{\text {part }}$, the deduced number of participants.

in the form of Fig. 1 would be equal to the $p-\bar{p}$ results, which they clearly are not. In fact this figure illustrates the range of validity of the WNM, if in your mind's eye you extend the $p-\bar{p}$ line to 0.9 at the left edge of the figure. The only nuclear collision exactly on the line is the $\mathrm{NA} 49 \mathrm{~Pb}+\mathrm{Pb}$ measurement at $\sqrt{s_{N N}}=10 \mathrm{GeV}$. The AGS measurements fall below the line, smaller multiplicities than the WNM, while for $\sqrt{s_{N N}} \geq 20 \mathrm{GeV}$, the $\mathrm{A}+\mathrm{A}$ measurements are above the WNM. The WNM works well only at CERN/FNAL fixed target energies, $\sqrt{s_{N N}}=10-20 \mathrm{GeV}$, but this has had major influence on the field since these data have been available for over 25 years. ${ }^{5}$ Also, proportionality to the number of participants is the only possibility in simple thermodynamic models, since volume is the only available 'extensive' variable.

PHOBOS also meaures the pseudo-rapidity dependence of the charged particle multiplicity, $d n_{c h}(\eta) / d \eta /\left(0.5 N_{\text {part }}\right)$, Fig 1 (right). The distributions for all 3 values of centrality shown exhibit a clear rapidity plateau, nearly \pm 2 units wide, followed by a rapid drop-off towards larger pseudorapidities, with a fwhm of nearly 7 units. The total charged multiplicity is $\sim 4000 \pm 10 \%$ for the most central collisions. On the falling edges of the distribution the curves all cross, indicating that the WNM works in the projectile and target fragmentation regions but not on the central plateau. Also, the width of the distribution decreases for more central collisions. 

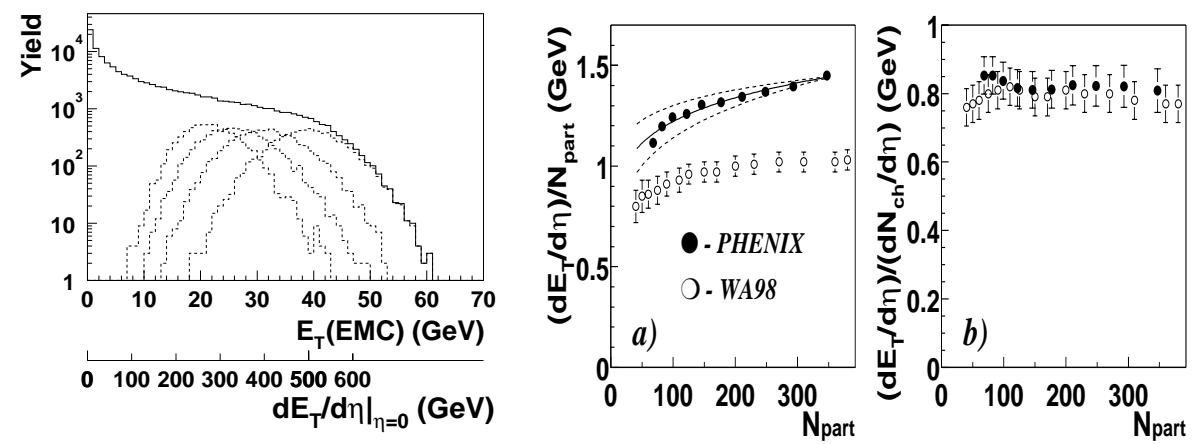

Figure 2. (left)Mid-rapidity $d E_{T} /\left.d \eta\right|_{\text {mid }}$ distribution from PHENIX in a solid angle $|\eta|<$ $0.38, \Delta \phi=44.4^{\circ}$, corrected to $2 \pi$ in azimuth (solid curve). Dotted curves represent semi-inclusive $d E_{T} /\left.d \eta\right|_{\text {mid }}$ distributions for the 4 topmost $5 \%$ slices of the of the reference distribution, corresponding to 211, 248, 293 and 347 participants. (right) a) Average mid-rapidity transverse energy per participant in PHENIX $\left(\sqrt{s_{N N}}=130 \mathrm{GeV}\right)$ compared to WA98 $\left(\sqrt{s_{N N}}=17.2 \mathrm{GeV}\right)$; b) Average transverse energy per charged particle versus the number of participants.

\section{2 $E_{T}$-Transverse Energy}

The mid-rapidity transverse energy distribution, $d E_{T} /\left.d \eta\right|_{\text {mid }}$, where $E_{T} \equiv$ $\sum_{i} E_{i} \times \sin \theta_{i}$ for all particles, charged and neutral, is shown on Fig. 2 (left), as measured by PHENIX. ${ }^{7}$ The shape of the distribution (solid line) exhibits the nuclear geometry, as confirmed by the dotted curves which represent the $E_{T}$ distributions as a function of centrality - more central, more overlap of the nuclei, more $E_{T}$. The detailed shape of the upper edges of the distributions depends, in addition, on the solid angle of the measurement-flatter for a small solid angle (as shown) and steeper for a larger acceptance. The centrality dependence of $d E_{T} /\left.d \eta\right|_{\text {mid }}$ per participant is shown on Fig. 2 (right). A clear increase with centrality, beyond the dotted systematic errors, is evident for the RHIC data, again showing the failure of the WNM, compared to the CERN data (WA98) where there is no change above 100 participants. It is also clear that the $d E_{T} /\left.d \eta\right|_{\text {mid }}$ at RHIC is $>40 \%$ higher than at CERN for the most central collisions. (The Bjorken energy density at RHIC ${ }^{7}$ is estimated as $\epsilon_{B j}=4.6 \mathrm{GeV} / \mathrm{fm}^{3}$.) By contrast, the mean $E_{T}$ per charged particle at both c.m. energies is constant versus centrality, at the same value $0.8 \mathrm{GeV}$, indicating that the additional energy density at RHIC is achieved mainly by an increase in particle production rather than by an increase of

qcd2001proc: submitted to World Scientific on September 25, 2001 
average transverse energy per particle. The evolution of the $\left\langle p_{T}\right\rangle$ from CERN to RHIC energies is further addressed in the next section.

\section{Identified Charged Particles}

The systematics of the mid-rapidity $p_{T}$ distribution of identified charged particles $\left(\pi^{ \pm}, K^{ \pm}, p^{ \pm}\right)$at RHIC is measured in the PHENIX experiment (Fig. 3). ${ }^{8}$ The excellent particle identification is obtained by precison Time of Flight
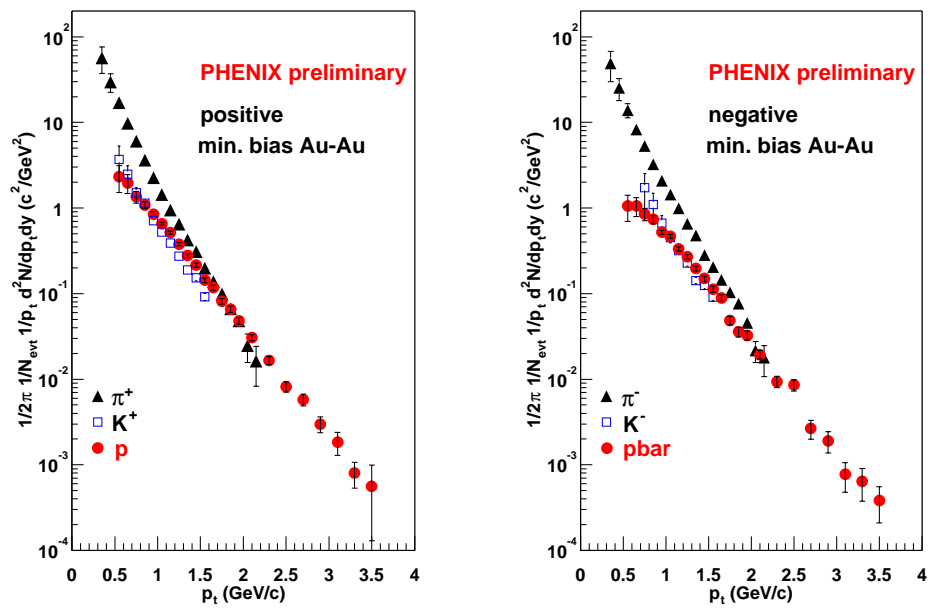

Figure 3. Semi-Inclusive Invariant Yield for Min-Bias Au+Au

(TOF) covering the solid angle $|\eta|<0.35, \Delta \phi=\pi / 4$, see Fig. 4 (left). All four RHIC experiments agree on the relative abundances, $\bar{p} / p \sim 0.65$, $K^{-} / K^{+} \sim 0.9, \pi^{-} / \pi^{+} \sim 1$, for minimum bias or more central collisions. The large increase of $K^{-} / K^{+}$, and $\bar{p} / p$ from CERN (AGS) $\mathrm{Pb}+\mathrm{Pb}(\mathrm{Au}+\mathrm{Au})$ collisions, probably reflects the similar increase with $\sqrt{s}$ in $p-p$ collisions; but detailed differences remain to be investigated. It is also clear from Fig. 3 that the particles and antiparticles exhibit essentially identical $p_{T}$ spectral shapes and that the steepness of the $p_{T}$ distributions decreases with increasing mass. This can be seen in finer detail in Fig. 4 (right) where $\left\langle p_{T}\right\rangle$ remains constant for all species, for $N_{\text {part }} \geq 150$, at values that are successively larger in ratio to the $\bar{p}-p$ value with increasing mass. These trends are quite similar to lower energy (AGS) $\mathrm{Au}+\mathrm{Au}$ data ${ }^{9}$, and the values for central collisions at RHIC are only $\sim 10 \%$ larger than at the AGS. Also the values of $\left\langle E_{T}\right\rangle /\left\langle n_{c h}\right\rangle$ vs

qcd2001proc: submitted to World Scientific on September 25, 2001 

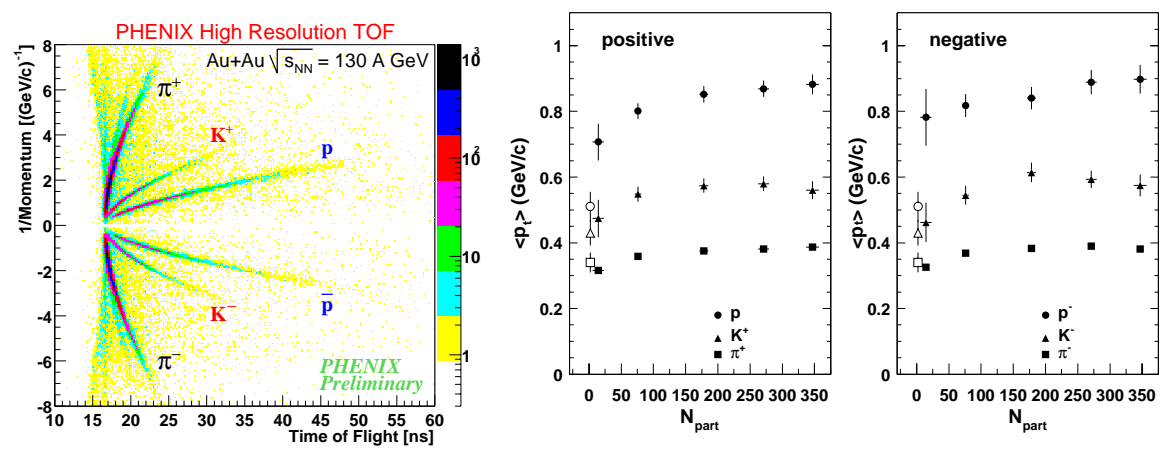

Figure 4. (left)PHENIX Particle I.D. by Time of Flight; (right) Filled points: $\left\langle p_{T}\right\rangle$ vs $N_{\text {part }}$, Open points: $\bar{p}-p$ and $p-p$ data interpolated to $\sqrt{s}=130 \mathrm{GeV}$.

$N_{\text {part }}$ derived from these data are in excellent agreement with Fig. 2. Apart from the relative increase in the production of $K^{-}$and $\bar{p}$ with increasing $\sqrt{s}$, there are no dramatic differences in $p_{T}$ spectra or $\left\langle p_{T}\right\rangle$ vs. centrality from AGS to RHIC energies.

\section{Flow}

\subsection{Correlation to the reaction plane}

An intriguing feature of Heavy Ion collisions is flow, pictured as the collective (rather than thermal) motion of particles outwards from the collision. Flow comes in at least 3 different varieties. At low energies, $E \leq 1 A \mathrm{GeV}$, where there is very little particle production, the participating nucleons all scatter away from other the nucleus due to the high nuclear compressibility. This is called directed flow and results in a scattering plane with an anisotropic particle distribution such that e.g. all participants move to the left in the projectile region and to the right in the target region. Also, the slow moving spectators block the produced particles so that they 'squeeze' out perpendicular to the scattering plane. ${ }^{10}$ At AGS energies, the spectators move faster and more particles are produced, so that for $E>4 \mathrm{AGeV}$ there is a transition to where particles are produced dominantly in the production plane outwards from the 'almond'. ${ }^{11}$ This 'elliptic' flow is left-right symmetric, and gives a $\cos (2 \phi)$ distribution of particles azimuthally around the reaction plane. Both these two 'anisotropic' flows vanish for central collisions, where yet another type of

qcd2001proc: submitted to World Scientific on September 25, 2001 
flow, called transverse or radial, occurs. This is thought to be the cause of the increase of $\left\langle p_{T}\right\rangle$ with particle mass shown in Fig. 4.

A spectacular result on elliptic flow at RHIC was obtained by STAR, see
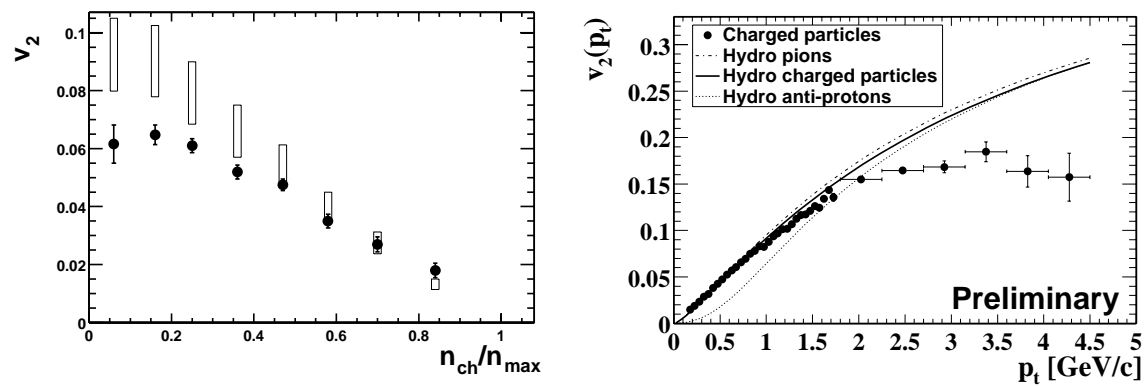

Figure 5. STAR Elliptic flow: (left) $v_{2}$ vs centrality (solid points) open boxes are proportional to the eccentricity of the 'almond'; (right) $v_{2}\left(p_{T}\right)$ for minimum bias collisions.

Fig. 5. ${ }^{12}$ The elliptic flow, measured by $v_{2}=\langle\cos (2 \phi)\rangle$, tracks the eccentricity of the 'almond' very well, apparently indicating a collective transfer of the original distribution of the 'almond' in position space to the particle directions in momentum space. The peak value of $v_{2}$ increases from $\sim 2 \%$ at AGS energies to $\sim 4 \%$ at CERN energies to $\sim 6 \%$ at RHIC. ${ }^{12}$ The $p_{T}$ dependence of $v_{2}$ for min-bias collisions is also very interesting, see Fig. 5 (right): $v_{2}$ increases with $p_{T}$ up to $2 \mathrm{GeV} / \mathrm{c}$, apparently following expectations of hydrodynamics, but then seems to plateau above $2 \mathrm{GeV} / \mathrm{c}$.

\subsection{2-particle correlations - flow and interferometry}

PHENIX 13 measures elliptic flow using 2-particle correlations, where $v_{2}^{2}=\left\langle\cos 2\left(\phi_{1}-\phi_{2}\right)\right\rangle$, a much smaller effect, and obtains results in agreement with the reaction plane analysis. ${ }^{12}$

STAR has measured $2-\pi^{-}$interferometry correlations, ${ }^{14}$ which measure the size of the emitting source of particle production. These show a smooth continuation from AGS and CERN energies to RHIC. No anomalous sizes due to e.g. a long lived mixed phase of hadronic matter and Quark Gluon Plasma have been seen. However, the dependence of the radii on the $\left\langle p_{T}\right\rangle$ of the $\pi^{-}$-pair is stronger at RHIC than at lower energies and is considered to be quite interesting. 


\section{$5 \quad$ High $p_{T}$ Physics}

In $p-p$ and $\bar{p}-p$ collisions, particle production at mid-rapidity has an exponential tail for $p_{T} \leq 2 \mathrm{GeV} / \mathrm{c}$, which depends very little on $\sqrt{s}$, and a power-law tail for $p_{T} \geq 2 \mathrm{GeV} / \mathrm{c}$ which varies systematically with $\sqrt{s}$. In this high $p_{T}$ region, the particles are the fragments of jets which result from the 2-body hard-scattering of the constituent quarks and gluons of the nucleon. ${ }^{15,16}$ Hard-scattering is a point-like process, with excellent PQCD predictions $\sim 10 \%$ for $p-p$ and $\bar{p}-p$ collisions. For $\mathrm{p}+\mathrm{A}$ or $\mathrm{A}+\mathrm{A}$ collisions the inclusive cross sections should scale by the number of point sources, $A$ for $\mathrm{p}+\mathrm{A}$ or $A^{2}$ for $\mathrm{A}+\mathrm{A}$ collisions. As a function of impact parameter, the semi-inclusive yield scales by the average nuclear overlap integral $\left\langle T_{A B}\right\rangle$ for the selected centrality class.
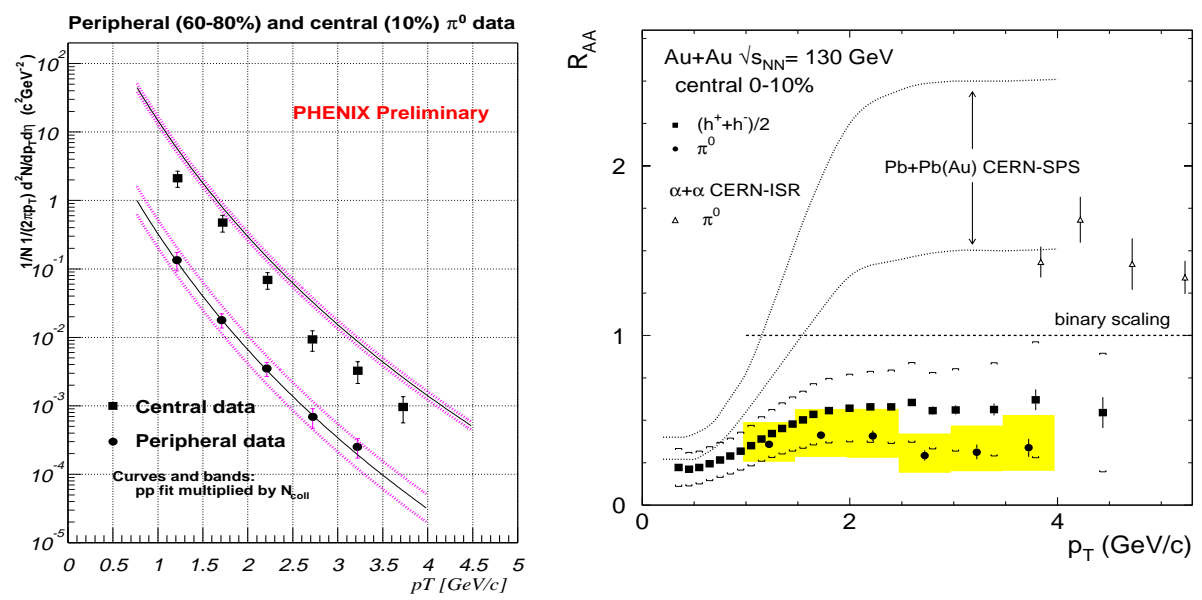

Figure 6. (left) PHENIX semi-inclusive invariant yield of $\pi^{0}$ vs $p_{T}$ for central and peripheral collisions, compared to the pointlike scaling of $p-p$ measurements; (right) $R_{A A}$, the ratio of the measured charged hadron and $\pi^{0} p_{T}$ spectra to the pointlike scaled $p-p$ data for central collisions compared to lower energy A+A measurements illustrated by data points from $\alpha-\alpha$ at the CERN ISR and by a band representing the CERN-SPS Pb+Pb measurements.

PHENIX has measured the semi-inclusive invariant $p_{T}$ spectra of nonidentified charged hadrons and identified $\pi^{0}$ up to $4-5 \mathrm{GeV} / \mathrm{c}$ for both peripheral and central collisions, see Fig. $6 .{ }^{17}$ Peripheral collision spectra agree with the pointlike scaling of $p-p$ and $\bar{p}-p$ measurements, while central collisions show a deficit compared to pointlike scaling. This is further em-

qcd2001proc: submitted to World Scientific on September 25, 2001 
phasized in Fig. 6 (left) where the ratio $R_{A A}$ of the central $\mathrm{A}+\mathrm{A}$ data to the pointlike scaled $p-p$ measurements is shown. The deficit of high $p_{T}$ particles, $R_{A A}<1$, has never been seen before in $\mathrm{p}+\mathrm{A}$ or $\mathrm{A}+\mathrm{A}$ collisions - at lower energies the yield is enhanced over pointlike scaling, known as 'the Cronin effect' (as shown). Could this deficit at RHIC be evidence of energy loss of the outgoing hard-scattered partons in a hot, dense, deconfined medium at

RHIC, as I hoped at a previous workshop in this series? ${ }^{16}$ I guess that we'll have to wait for the next workshop to find out.

\section{References}

1. See papers by A. Denisov, J. Katzy and S. N. White in reference ${ }^{2}$.

2. Proceedings of Quark Matter 2001, to be published in Nucl. Phys. A. See also http://www.rhic.bnl.gov/qm2001.

3. B. B. Back, et al, PHOBOS Collab., Phys. Rev. Lett. 85, 3100 (2000).

4. B. B. Back, et al, PHOBOS Collab., nucl-ex/0108009, submitted to Phys. Rev. Letters.

5. W. Busza, et al, Phys. Rev. Lett. 34, 836 (1975); J. E. Elias, et al, Phys. Rev. D22, 13 (1980).

6. A. Bialas, A. Bleszynski and W. Czyż, Nucl. Phys. B111, 461 (1976).

7. K. Adcox, et al, PHENIX Collab., Phys. Rev. Lett. 87, 052301 (2001).

8. J. Velkovska, et al, PHENIX Collab., nucl-ex/0105012 in reference ${ }^{2}$.

9. L. Ahle, et al, E802 Collab., Phys. Rev. C57, R466 (1998); Y. Akiba, et al E802 Collab., Nucl. Phys. A610, 139c (1996).

10. H. A. Gustafsson, et al, Phys. Rev. Lett. 52, 1590 (1984).

11. C. Pinkenburg, et al, E895 Collab., Phys. Rev. Lett. 83, 1295 (1999).

12. K. H. Ackermann, et al, STAR Collab., Phys. Rev. Lett. 86, 402 (2001); see also R. J. M. Snellings, et al, nucl-ex/0104006 in reference ${ }^{2}$.

13. R. A. Lacey, et al, PHENIX Collab., nucl-ex/0105003 in reference ${ }^{2}$.

14. C. Adler, et al, STAR Collab., Phys. Rev. Lett. 87, 082301 (2001).

15. J. F. Owens, Rev. Mod. Phys. 59, 465 (1987)

16. M. J. Tannenbaum, pp 280-285 in Proc. IV Workshop on QCD, Eds. H. M. Fried, B. Müller (World Scientific, Singapore, 1998).

17. K. Adcox, et al, PHENIX Collab., nucl-ex/0109003, submitted to Phys. Rev. Letters. 